\title{
Exercise stress testing as the significant clinical modality for management of hypertension
}

\author{
Bonpei Takase \\ Hypertension Research (2012) 35, 706-707; doi:10.1038/hr.2012.47; published online 12 April 2012
}

$\mathrm{T}$ he response of blood pressure during exercise stress testing is an important evaluation parameter when such testing is used as a clinical diagnostic modality. Several studies have associated the blood pressure response with the severity of hypertension or the likelihood of undiagnosed hypertension in patients, who are at risk for essential hypertension. ${ }^{1}$ However, exercise stress testing using a treadmill is usually used to diagnose and/or assess the effects of treatment for coronary artery disease. Twelve-lead electrocardiography, heart rate and blood pressure responses and exercise tolerance are typical measures for evaluating exercise stress testing. The criteria for clinically significant electrocardiographic changes, for example, ST segment changes, have been defined and are routinely applied in the clinical management of coronary artery disease. Heart rate responses including chronotropic incompetence defined by Ellestad ${ }^{2}$ have been established. Normal values for heart rate responses to exercise are known as 'the predicted maximal heart rate by age'. Heart rate and blood pressure responses are frequently used as parameters for predicting myocardial oxygen consumption as non-invasive indices; the heart rate and double product are calculated by heart rate multiplied by blood pressure during exercise stress testing. The normal ranges of blood pressure response to exercise stress testing are as shown in Figure $1 .^{3}$ Normal systolic and diastolic responses to exercise stress testing should not exceed 220 and $100 \mathrm{~mm} \mathrm{Hg}$, respectively. Systolic blood

Dr B Takase is at the Department of Intensive Care Medicine, National Defense Medical College, Tokorozawa, Saitama, Japan.

E-mail: bonpeit@ndmc.ac.jp. pressure of $>230 \mathrm{mmHg}$ is generally considered hazardous. According to the recommended guidelines for exercise stress testing, elevations in either systolic and/or diastolic blood pressure to 250 and $130 \mathrm{~mm} \mathrm{Hg}$, respectively, are indications to terminate the test.

Lima et al. ${ }^{4}$ reported in this issue of Hypertension Research that age and body mass index (BMI) are significant predictors for an exaggerated blood pressure response (EBPR) during exercise stress testing. An EBPR is defined as an increase in delta systolic blood pressure of $\geqslant 7.5 \mathrm{~mm} \mathrm{Hg} /$ METS (metabolic equivalents) and/or systolic blood pressure at the peak of the exercise exceeding $220 \mathrm{~mm} \mathrm{Hg}$ or a delta diastolic blood pressure increase of $\geqslant 15 \mathrm{~mm} \mathrm{Hg}$ compared with resting values. They also studied associations between EBPR and insertion/deletion polymorphisms of angiotensin-converting enzyme and M235T of angiotensinogen. However, they found no significant relationships. Others have found that the exercise-induced hypertension expressed as EBPR by Lima et al. ${ }^{4}$ correlates with subsequent cardiac events ${ }^{5}$ in patients with cardiovascular diseases. In addition, a hypertensive blood pressure response to exercise in healthy individuals can predict the development of hypertension. ${ }^{6}$ These results suggest that changes in blood pressure during exercise are associated with the pathophysiology of hypertension. Thus, the findings of Lima et al., in which clinical parameters such as age and BMI are significantly indicated for predicting EBPR, are notable and useful for interpreting results of routine exercise stress tests.

Age and BMI are both associated with sympathetic activity and peripheral vascular resistance during exercise stress testing.
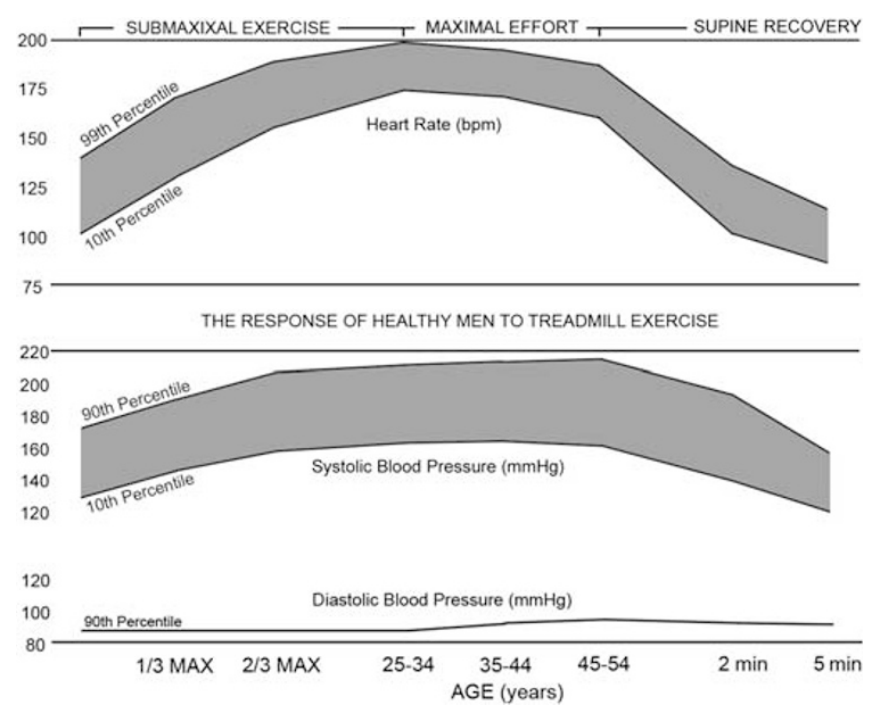

Figure 1 Normal physiological responses to submaximal and maximal exercise treadmill testing derived from apparently healthy men aged 24-54 years old (adopted from Wolthuis et al. ${ }^{3}$ ). 
Table 1 Cumulative mean increase in systolic blood pressure $(\mathrm{mm} \mathrm{Hg})$ in response to work load progress (expressed as METS (metabolic equivalents) during exercise testing) in healthy men (adopted from Fox and Naughton ${ }^{10}$ )

\begin{tabular}{|c|c|c|c|c|c|c|c|}
\hline \multirow{2}{*}{ Age range } & \multicolumn{7}{|c|}{ Work load progress expressed as METS } \\
\hline & 4 & 5 & 6 & 7 & 8 & 9 & 10 \\
\hline $20-29$ & 25 & 32 & 36 & 39 & 47 & 51 & 59 \\
\hline $30-29$ & 25 & 32 & 40 & 45 & 51 & 61 & 61 \\
\hline $40-49$ & 26 & 38 & 43 & 47 & 54 & 57 & 67 \\
\hline $50-59$ & 29 & 41 & 48 & 58 & 64 & 71 & 79 \\
\hline Total group & 26 & 35 & 42 & 47 & 54 & 60 & 66 \\
\hline
\end{tabular}

(Unit in each cell, $\mathrm{mm} \mathrm{Hg}$; cumulative mean systolic blood pressure increase)

Reports indicate that the main mechanism for exercise-induced hypertension or EBPR is a failure to reduce peripheral vascular resistance during exercise. ${ }^{6}$ Vasodilator capacity is impaired in the skeletal muscle of hypertensive patients during exercise. ${ }^{7}$ Many other factors that regulate peripheral vascular tone during exercise have also been reported. Among them, sympathetic tone is most important for determining peripheral vascular resistance. ${ }^{8}$ Aging might increase peripheral vascular tone during exercise through increasing sympathetic activity. ${ }^{8}$ In addition, aging itself could increase peripheral vascular tone through impairing endothelial function that is also a reported determinant of exercise tolerance in humans. ${ }^{9}$ An increased BMI is a sign of obesity and the combination of obesity, mild hypertension, glucose intolerance and dyslipidemia is the typical clinical presentation of metabolic syndrome. Sympathetic tone is generally more activated in individuals with, than without, metabolic syndrome. Thus, the findings of Lima et al. ${ }^{4}$ in which age and BMI are significantly associated with exercise-induced hypertension or EBPR are logical and rational. Table 1 shows that the cumulative increase in mean systolic blood pressure among older adult men is larger in response to a work load expressed as a METS increase (work load progress). ${ }^{10}$

Exercise stress testing is mostly considered as a typical tool for diagnosing coronary artery disease or determining exercise capacity. Exercise stress testing is frequently applied during exercise rehabilitation programs for patients with cardiovascular disorders. If the clinical meaning of exerciseinduced hypertension or EBPR could be understood in more detail, exercise stress testing could be used as a clinical tool for evaluating hypertensive patients and/or individuals at high risk of developing further hypertension in the near future. Furthermore, exercise stress testing might be used for evaluating the effects of anti-hypertensive therapies. Prospective clinical studies should be performed, such as follow-up of hypertensive patients and healthy individuals with EBPR, to determine the significance of EBPR for predicting the development of organ damage or untoward outcomes. From this aspect, the recent report by Lima et al. ${ }^{4}$ published in the Journal of 'Hypertension Research' is very valuable.

In contrast, a significant relationship between EBPR and insertion/deletion polymorphisms of angiotensin-converting enzyme or M235T of angiotensinogen has not been identified. As these polymorphisms are established as significant factors in the pathogenesis of hypertension, the role of polymorphisms in EBPR should be further investigated in the near future.

\section{CONFLICT OF INTEREST}

The authors declare no conflict of interest.

1 Allison TG, Cordeiro MA, Miller TD, Daida H, Squires RW, Gau GT. Prognostic significance of exercise-induced systemic hypertension in healthy subjects. Am J Cardiol 1999; 83: 371-375.

2 Ellestad MH, Wan MK. Predictive implications of stress testing. Follow-up of 2700 subjects after maximum treadmill stress testing. Circulation 1975; 51: 363-369.

3 Wolthuis RA, Froelicher Jr VF, Fischer J, Triebwasser JH. The response of healthy men to treadmill exercise. Circulation 1977; 55: 153-157.

4 de Lima SG, de Albuquerque MFPM, de Oliveira JRM, Ayrers CFJ, da Cunha JEG, de Oliveira DF, de Lemos RR, de Souza MBR, e Silva OB. Exaggerated blood pressure response during exercise treadmill testing: functional and hemodynamic features, and risk factors. Hypertens Res 2012; 35: 733-738.

5 Miyai N, Arita M, Miyashita K, Morioka I, Shiraishi T, Nishio I. Blood pressure response to heart rate during exercise test and risk of future hypertension. Hypertension 2002; 39: 761-766.

6 Palatini P. Exaggerated blood pressure response to exercise: pathophysiologic mechanisms and clinical relevance. J Sports Med Phys Fitness 1998; 38: 1-9.

7 Bond Jr V, Franks BD, Tearney RJ, Wood B, Melendez $M A$, Johnson L, lyriboz Y, Bassett Jr DR. Exercise blood pressure response and skeletal muscle vasodilator capacity in normotensives with positive and negative family history of hypertension. J Hypertens 1994; 12: 285-290.

8 Hashimoto I, Miyamura M, Saito M. Initiation of increase in muscle sympathetic nerve activity delay during maximal voluntary contraction. Acta Physiol Scand 1998; 164: 293-297.

9 Takase B, Uehata A, Fujioka T, Kondo T, Nishioka T, Isojima K, Satomura K, Ohsuzu F, Kurita A. Endothelial dysfunction and decreased exercise tolerance in interferon-alpha therapy in chronic hepatitis C: relation between exercise hyperemia and endothelial function. Clin Cardiol 2001; 24: 286-290.

10 Fox 3rd SM, Naughton JP. Physical activity and the prevention of coronary heart disease. Prev Med 1972; 1: 92-120. 